\title{
Multi-axis Stiffness Sensing Device for Medical Palpation
}

\author{
A. Faragasso, A. Stilli, J. Bimbo, H.A. Wurdemann, K. Althoefer, Member, IEEE
}

\begin{abstract}
This paper presents an innovative hand-held device able to compute stiffness when interacting with a soft object. The device is composed of four linear indenters and a USB camera. The stiffness is computed in real-time, tracking the movements of spherical features in the image of the camera. Those movements relate to the movements of the four indenters when interacting with a soft surface. Since the indenters are connected to springs with different spring constants, the amount they move is different from the amount the others move when contacting a soft object. The proposed multi-indenting device allows measuring the object's stiffness as well as the pan and tilt angles between the sensor and the surface of the soft object. Tests were performed to evaluate the accuracy of the proposed palpation mechanism against commercial springs of known stiffness. Results show that the accuracy and sensitivity of the proposed device increases with the softness of the examined object. Preliminary tests with silicon show the ability of the sensing mechanism to characterize phantom soft tissue for small indentation. It is noted that the results are not affected by the orientation of the device when probing the surface. The proposed sensing device can be used in different applications, such as external palpation for diagnosis or, if miniaturised, embedded on an endoscopic camera and used in Minimally Invasive Surgery (MIS).
\end{abstract}

\section{INTRODUCTION}

Palpation is a medical diagnosis technique in which texture, size, consistency and location of soft tissue areas are examined by clinicians using vision combined with the sensation received from their finger tips when touching soft tissue. During the physical procedure, clinicians press their fingers around the relevant area to determine its characteristics according to indentation depth (how much they deform tissue) and the reaction force they feel. This information is then used for diagnosis. Palpation can be performed with a single hand or two hands on the skin or on internal soft tissue of the body during surgeries in a light or deep way. Light palpation is performed to detect tenderness, muscle spasm, or rigidity of the abdomen and to evaluate abnormal lesions in the breast, the prostate gland, the lymph nodes, or the testes. If pain is present, gentle palpation begins farthest away from the pain to localise the point of maximum tenderness.

Deep palpation is used to determine the size of the liver,

*The work described in this paper is partially funded by the Seventh Framework Programme of the European Commission under grant agreement 287728 in the framework of EU project STIFF-FLOP, as well as by the National Institute for Health Research (NIHR) Biomedical Research Centre based at Guy's and St Thomas' NHS Foundation Trust and King's College London. The views expressed are those of the authors and not necessarily those of the NHS, the NIHR or the Department of Health.

A. Faragasso, A. Stilli, J. Bimbo, H.A. Wurdemann and K. Althoefer are with the Centre for Robotics Research, Department of Informatics, King's College London, London, WC2R 2LS, UK angela.faragasso, agostino.stilli, joao.bimbo, helge.wurdemann, k.althoefer@kcl.ac.uk

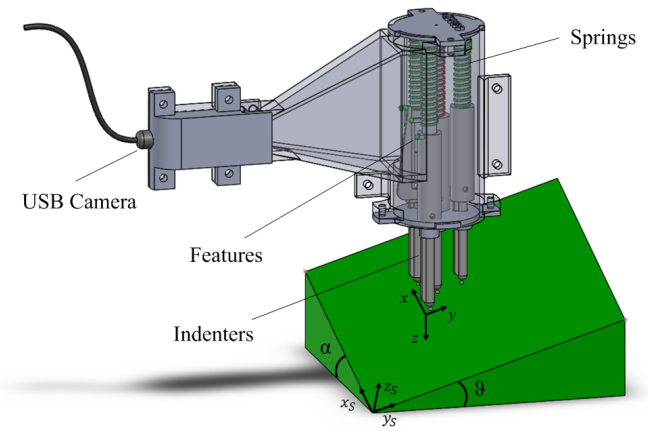

Fig. 1: Multi-axis stiffness sensor interacting with a soft surface. The pan and tilt angles between the sensing device and the surface of the soft object are $\alpha$ and $\theta$ respectively.

spleen, or kidneys and to detect an abnormal mass [1] [2]. Nowadays, palpation is a common medical routine used in different contexts and scopes. For instance, a proper breast examination includes frequent (at least monthly) selfexaminations and an annual examination by a clinician. Palpation should be methodical and performed over the entire breast. Suspicious breast lesions are hard and fixed rather than movable. Skin retraction or breast asymmetry can indicate an underlying, potentially serious lesion. Cancers are usually not tender, and benign lesions are more likely to be round, elastic or firm, movable, and well-defined. Similarly, suspicious prostate lesions are hard irregular nodules, whereas benign prostatic hyperplasia (BPH) is a soft symmetrical enlargement of the gland [1].

Palpation is also performed during surgeries. In open surgery, doctors rely on the sense of touch by their fingers, on their vision and experience to identify the edges of hidden tumours and to locate hidden blood vessels and other anatomical structures, i.e. during external invasive palpation procedures [3].

Currently, it is not possible to carry out diagnosis using palpation in Minimally Invasive Surgery (MIS) where surgeons work with laparoscopic equipment and instruments with up to $10 \mathrm{~mm}$ diameter including endoscopic cameras and hand-held devices that fit through small incisions called trocar ports. It is proved, however, that in-situ estimation of soft tissue's mechanical property may improve the quality of MIS [4] [5]. In current MIS procedures without soft tissue stiffness measurement systems, palpation is subjective as a means to diagnose and is heavily dependent on the medical doctor's experience and thus can be challenging for an inexperienced surgeon. As such, surgical devices able to compute tissue properties can greatly improve the quality of diagnosis and help training young surgeons. Despite all attempts and studies in sensing technologies for soft tissue 
characterisation, a real-time system able to determine the stiffness of the anatomical area is still missing and is highly desirable [5]. Such systems could also be used as a means to do self-diagnosis at home.

In this paper, we propose a vision-based multi-directional stiffness sensor as shown in Fig 1. The paper is organised as follows: Section II reviews the current State of the Art in stiffness sensing technologies. The design of the proposed sensing prototype is described in Section III Section IV presents the real-time vision processing algorithm. The mathematical model derived and the optimisation algorithm are presented in Section $\mathrm{V}$ ] The experimental results are reported in Section VI.

\section{BACKGROUND}

The understanding of the mechanical properties of soft tissue is important to increase the quality of the diagnosis. Novel medical practices use sophisticated diagnostic tests commonly using Computer Tomography (CT), Ultrasound imaging (US) and Magnetic Resonance Imaging (MRI) [6] [7]. However, these tests cannot provide directly a measure of tissue elasticity.

Frei et al. [8] proposed an instrument for breast examination using a plurality of spaced piezoelectric force sensors. The thickness of very small tissue region were computed evaluating difference in the output of adjacent transducers. In [9], authors developed a tactile sensing instrument called the Tactile Tumour Detector (TTD). The main parts of this device are a tactile probe, an electrical circuit, a data processor and a tactile display. Although the device is able to detect abnormal objects, it works only with specific simulated models and cannot be used in real-time. Mechanical Imaging (MI) is a new modality of medical diagnostics that is based on visualising the sense of touch. In MI, the internal structures of an organ are shown by measuring the pattern of mechanical stresses on its surface. MI is simple, fast, inexpensive and safe. However, MI methods are general and have much broader implications in a variety of diagnostic and surgical applications that require cancer diagnosis, assessment and localisation of abnormal tissue for biopsy and characterisation [10].

In the context of MIS, stiffness measurement approaches become even more problematic. During the past years, researchers have proposed different systems to measure forces and tactile information and feed this back to the surgeon during the operation [11] [12]. Surgical instruments have been explored for different palpation approaches, such as indenting or grasping soft tissue [13]. Most of the MISrelated research studies about soft tissue characterisation are using the Nano-17 (ATI, Industrial Automation), a sterilisable commercial 6 DoF sensor system with a diameter of $17 \mathrm{~mm}$, [14] [15]. However, constraints on size, geometry, costs, bio-compatibility and sterilisability make some of these approaches unsuitable for MIS. So far, tactile and force sensors have been applied to surgical tools in MIS to measure local tissue properties. Providing this feedback supports to the surgeon operating with remote mechanisms [16] [17].

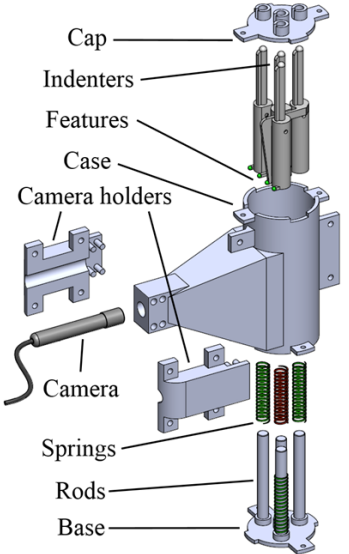

(a)

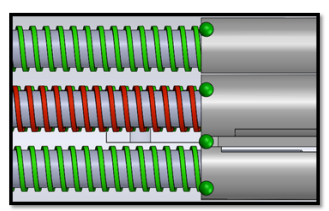

(b)

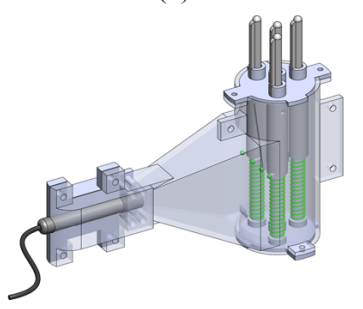

(c)
Fig. 2: CAD Drawing of the stiffness sensor in which the softer springs are represented in green and the stiffer in red: (a) Exploited View, (b) Camera View and (c) Assembled.

Research shows that a real solution is still missing and desirable [18].

In our previous paper [19], we proposed an uni-axial stiffness sensing device based on a real time visual tracker of linear movements of some spherical features [19]. We further developed our work and present an innovative multi-directional stiffness sensing device in this paper with the following features:

1) The presented device can compute the stiffness during palpation with soft tissue.

2) The computation of the stiffness does not depend on the inclination at which the surface is approached.

3) The sensing range and resolution can be easily modified by changing the distance between the camera, the spherical features and the springs used.

4) Our presented system has a simple mechanical structure which can be easily miniaturised. In this case, it can be used for internal diagnosis.

\section{Design of the Mechanical Sensor Device}

Exploded and assembled views of the stiffness sensor are shown in Fig. 2 The hand-held device is composed of four indenters, each of which is connected to a spring and a spherical feature, a standard USB camera and hosed in 3D printed sructure. The USB camera has an outer diameter of $7 \mathrm{~mm}$, a resolution of $640 \times 480$ and a frame rate of $30 \frac{\text { frames }}{\mathrm{s}}$. The body of the sensing device and the indenters have been manufactured with a high resolution 3D printer (HD-3000 Plus from 3D System) with an accuracy of $16 \mu \mathrm{m}$ along the $z$-axis and $42 \mu \mathrm{m}$ along the $x$-and $y$-axis) using ABS high density material. The used springs are made of SS316 steel, a material that is suitable for medical applications. Hence, the whole sensor has a relatively low cost since it has been created using commercially available components for less than $50 £$ in total and a structure produced from a $3 \mathrm{D}$ printer. Three of the springs have the same elastic constant, $0.05 \frac{\mathrm{N}}{\mathrm{mm}}$, the fourth has been chosen stiffer with a constant elastic value of $0.25 \frac{\mathrm{N}}{\mathrm{mm}}$. The movements of each indenter is coupled with the movement of a spring, hence 
the three indenters coupled with the same springs move with the same elastic constant resulting in a softer behaviour compared to the one related to the fourth indenter. As shown in the Fig. 2 the softer springs and their associated rods and indenters are arranged on a circle at $102^{\circ}$ intervals around the stiffer one placed in the center. When the sensing device is not interacting with an object surface the centres of the spherical features are in the same plane. The movements of each spherical feature is associated with the related indenter. The features are coloured (the green colour was chosen in this prototype) in order to create a clear contrast to the background and, hence to be easily tracked. During physical contact with the environment, the indenters slide over their respective rods leading to a compression of the four springs that generate, in turn, movement of the related spherical features. During the interaction the three softer get compressed differently when compared to the central one.

\section{Real-time Image Processing}

The pseudo code of the overall real-time image processing methodology is described in Algorithm 1. It shows the procedural steps of feature tracking, applying a Kalman filter and mapping the position of the tracked image centroids in the chosen reference frame. Each element is explained in more detail in the following subsections.

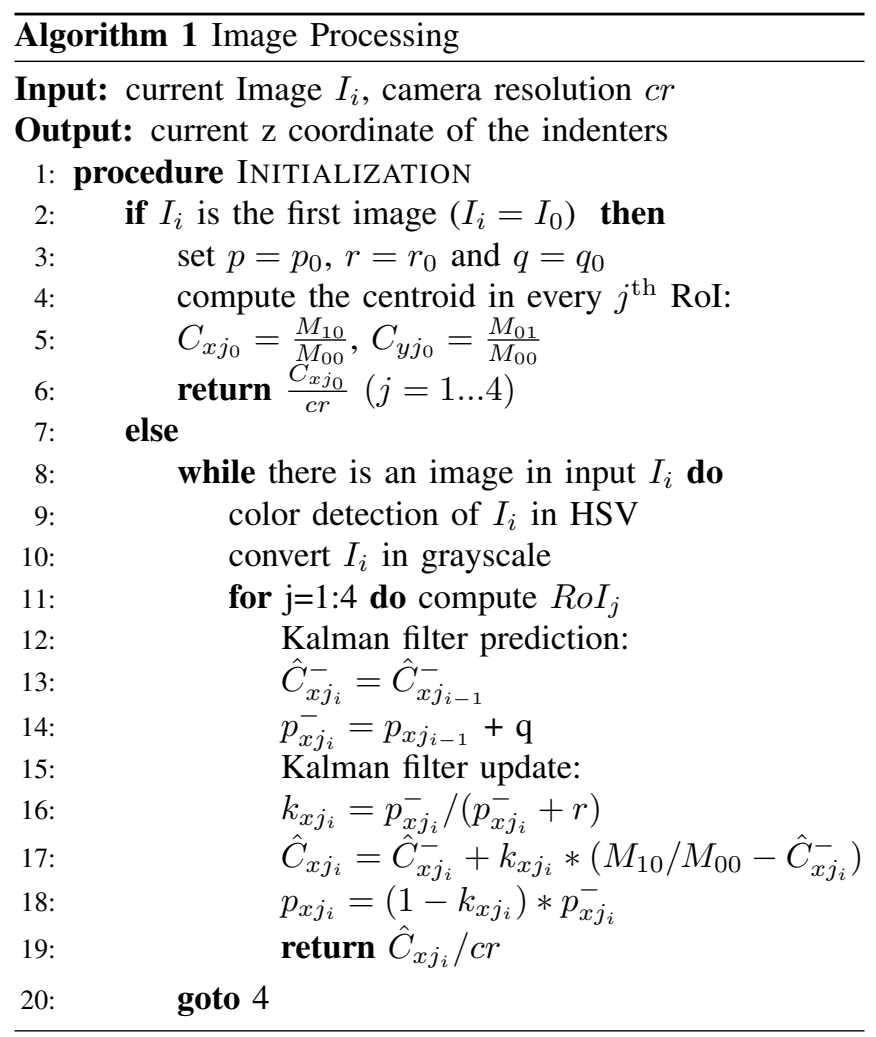

\section{A. Robust tracking of the spherical features}

The working principle of the proposed stiffness sensing device is based on the tracking of the spherical features which move with the sliding of the indenters during interaction with a soft object. In order to track the features with high accuracy, the ROS camera calibration tool was used to obtain the camera's intrinsic parameters and to produce images that are void of distortions of the lens. As presented in previous works [19] [20], the image processing algorithm here also uses the OpenCV library. The movement of the features is detected performing colour filtering in the HSV colour space. The camera image is divided into four vertical Regions of Interest (RoIs). Each of them is used to track a single feature. Unlike the previous work, here the centroids of the features are determined using the central moments on the grayscale images. In a filtered gray scale image with intensity $I(x, y)$, the raw image moments are defined as:

$$
M_{i, j}=\sum_{x} \sum_{y} x^{i} y^{j} I(x, y) .
$$

Using Equation (1), the position of the centroid in each RoI is expressed as:

$$
C_{x}=\frac{M_{10}}{M_{00}} \quad C_{y}=\frac{M_{01}}{M_{00}} .
$$

\section{B. One dimensional Kalman Filter on the image centroids}

The Kalman filter assumes that the state of a system at time $t$ evolved from the prior state at $t-1$ according to

$$
\mathbf{x}_{t}=\mathbf{A}_{t} \mathbf{x}_{t-1}+\mathbf{B}_{t} \mathbf{u}_{t}+\mathbf{w}_{t}
$$

where $\mathbf{x}_{t}$ is the state vector containing the terms of interest for the system at time $t, \mathbf{u}_{t}$ is the vector containing control inputs; $\mathbf{A}_{t}$ is the state transition matrix which applies the effect of each system state parameter at time $t-1$ on the system state at time $t ; \mathbf{B}_{t}$ is the control input matrix which applies the effect of each control input parameter in vector $\mathbf{u}_{t}$ on the state vector and $\mathbf{w}_{t}$ is the vector containing the process noise term for each parameter in the state vector. The process noise is assumed to be drawn from a zero mean multivariate normal distribution given by the covariance matrix $\mathbf{Q}_{t}$. Measurements of the system can also be performed according the following model:

$$
\mathbf{z}_{t}=\mathbf{H}_{t} \mathbf{x}_{t}+\mathbf{v}_{t},
$$

where $\mathbf{z}_{t}$ is the vector of measurements; $\mathbf{H}_{t}$ is the transformation matrix that maps the state vector parameters into measurement domain and $\mathbf{v}_{t}$ is the vector containing the measurement noise terms for each observation. The measurement noise is also assumed to be zero mean Gaussian white noise with covariance matrix $\mathbf{R}_{t}$. The main steps in the filter are the prediction, the innovation and the update. The Kalman filter is used to track the sliding of the spherical features along the $x$-axis. The filter is applied with each new measurement of the centroids and initialised with the process noise $q_{0}=0.09$, the sensor noise $r_{0}=4$ and the initial estimated error $p_{0}=6$. These values are chosen based on an emperical evaluation. $C_{x j_{0}}$ represents the $x$-coordinate of the four centroid in the corresponding $j^{\text {th }}$ RoI computed in the first image $I_{0}$ using Equation 2.

After the prediction step, the state of the filter is defined using $p, q, C_{x j_{i}}$ and the Kalman gain $k_{x j_{i}}$ as shown in Algorithm 1 . 


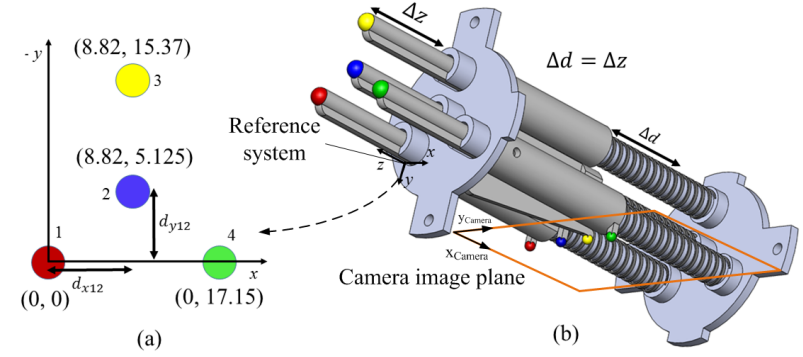

Fig. 3: Hand-held stiffness sensor: (a) the frontal view of the sensor showing the position of the indenters $(\mathrm{mm})$ with respect to the reference system. The indenters which are coupled to the corresponding spherical features are shown in (b). Each pair is represented by the same colour. When an indenter interacts with the surface of a soft object, it results in a displacement of $\Delta z$; the corresponding sphere moves by the same quantity, but appears in the camera images as a movement along the $x$-axis, hence, $\Delta x$.

\section{Mapping of the indenters movements}

To model the movements of the spherical features, a reference system has been chosen on the device as shown in Fig. 3(b). The position of the indenters are expressed in this defined reference system. The image plane is parallel to the $x$-z-plane of the reference system.

The three indenters are placed on the vertex of a triangle and one in its barycentre. During the interaction with a soft object, the indenters generate compression and decompression of the springs and, thus, produce linear movements of the spherical features within the image plane of the camera (see Fig. 3 b)). The resolution of the sensors is $12.7 \mathrm{~mm}$ per pixel, i.e. when the interaction with the soft surface causes a movement of the indenter of $12.7 \mathrm{~mm}$, the position of the correspondent centroid changes by one pixel. This coupled mechanism between the indenters and the planar movements of the spherical features allows computing the mapping between the variation in position of the centroids in the image, $\Delta x$, and the change in depth of the indenters $\Delta z$ as shown in Fig. 3 (b).

\section{Soft TISSUE Characterisation}

\section{A. Modelling soft tissue properties}

The mapping between the position of the spheres and the position of the indenters is used to compute the stiffness and the contact angles of probed surfaces. The sliding mechanism of the three indenters placed on the vertices of the triangle use the same type of spring, while the indenter in the center is connected to a spring with a higher spring constant, thus is moving less during the interaction with soft object than the other three. The stiffness of the surface in contact can be computed using the difference between the forces applied by the harder indenter placed in the barycentre of the triangle and the virtual force applied by the three "softer" indenters in the same point divided by the difference between those two [19]. The main improvement of our sensor device in comparison to the one presented in [19] is that the stiffness is computed solving a system of non linear equation and the resultant stiffness is independent of the contact angles. Seven parameters are used to describe a probing task: the palpation depth of the soft tissue caused by the interaction with the four indenters, the pan angle $\theta$, the tilt angle $\alpha$ and the stiffness of the tissue $K_{t}$ as shown in Fig. 11 The relation between these parameters is expressed by a system of non-linear equations $\mathbf{F}(\mathbf{x})=0$, where:

$$
\mathbf{F}(\mathbf{x})=\left\{\begin{array}{l}
d x_{1} \times K_{s}-K_{t} \times d t_{1} \\
d x_{2} \times K_{h}-K_{t} \times d t_{2} \\
d x_{3} \times K_{s}-K_{t} \times d t_{2} \\
d x_{4} \times K_{s}-K_{t} \times d t_{4} \\
d x_{2}+d t_{2}-d x_{1}-d t_{1}+\tan (\theta) \times d_{12 x}+\tan (\alpha) \times d_{12 y} \\
d x_{3}+d t_{3}-d x_{1}-d t_{1}+\tan (\theta) \times d_{13 x}+\tan (\alpha) \times d_{13 y} \\
d x_{4}+d t_{4}-d x_{1}-d t_{1}+\tan (\theta) \times d_{14 x}+\tan (\alpha) \times d_{14 y}
\end{array}\right.
$$

In Equation (5), $d x_{i}(i=1 \ldots 4)$ represents the position of the $i^{\text {th }}$ sphere, $d x_{i j}(i, j=1 \ldots 4)$ with $i \neq j$ represents the distance between the $i^{\text {th }}$ and $j^{\text {th }}$ sphere in the reference system of the sensor. $K_{s}$ and $K_{h}$ are the spring constants of the soft and hard springs, respectively. These two parameters are known. The unknown parameters are: the pan angle $\theta$, the tilt angle $\alpha$, the displacement of the soft tissue in the points of contact $d t_{i}$ with $(i=1 \ldots 4)$ and the stiffness of the soft surface $K_{t}$. The vector $\mathrm{x}$ contains the solution of the system of Equation (5) is found by solving a non-linear least squares problem.

\section{B. Stiffness Computation}

The Levenberg-Marquardt algorithm is applied here to solve Equation (5). This algorithm is an iterative optimisation technique that minimises the sum of squares of a set of nonlinear functions. It combines the advantages of gradientdescent and Gaussian-Newton methods [21]. If the reduction of the sum of squares at the current iteration is rapid, the algorithm behaves like a Gauss-Newton method; if an iteration gives insufficient reduction of the residual, it becomes a gradient-descent method which gradually converges [22]. This optimisation method is used to find the update rule of $x$ in Equation (5):

$$
\mathbf{x}_{k+1}=\mathbf{x}_{k}-\left(\mathbf{H}_{k}+\lambda_{k} \operatorname{diag}\left(\mathbf{H}_{k}\right)\right)^{\dagger} \mathbf{J}_{k}^{T} \mathbf{F}_{k},
$$

where $\mathbf{J}_{k}$ is the Jacobian matrix of $\mathbf{F}(\mathbf{x})$ evaluated at $\mathbf{x}_{k}$, $\mathbf{H}_{k} \approx \mathbf{J}_{\mathbf{k}}^{T} \mathbf{J}_{k}$ is an approximation of the Hessian matrix and $\lambda_{k}$ represents the non-negative damping factor that is adjusted at each iteration to interpolate between the gradient descent and the Newton's method. A high value for $\lambda$ favours the gradient descent, whereas a lower value favours Newton's method. Thus, $\lambda$ increases if $\left\|\mathbf{F}\left(\mathbf{x}_{k+1}\right)\right\|$ is greater than $\left\|\mathbf{F}\left(\mathbf{x}_{k}\right)\right\|$ and decreases otherwise. Our method converges when $\|\mathbf{F}(x)\|$ is less than a chosen threshold. Then, the current point $\mathbf{x}_{\mathbf{k}}$ is returned as the best-fit solution. In order to be able to find a solution to the unknown parameters and to compute the stiffness in real-time, we chose our threshold to be $\|\mathbf{F}(x)\|=0.0001$. In this case, the algorithm needs less than seven iteration to find a solution with high accuracy, as shown in Table [] 


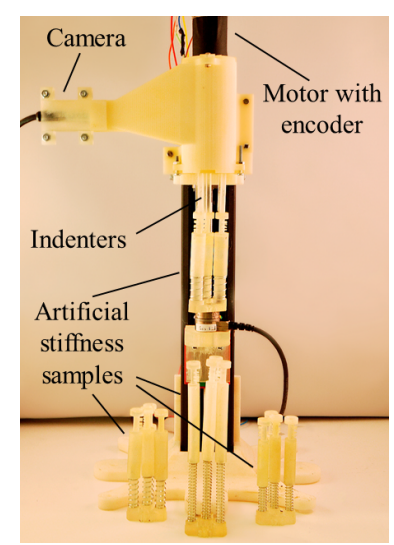

Fig. 4: Evaluation Setup

\section{EXPERIMENTAL RESUlTS}

A motorised linear module has been designed to evaluate the stiffness sensing device. Fig. 4 shows the setup of the benchmarking test rig. Different artificial stiffness samples have been created to simulate soft tissue [23]. These samples are composed of four linear rods with self-centering shaped heads. The movements of the samples are related to four springs with identical spring constants. Hence, this structure behaves like soft tissue with a known stiffness. The artificial stiffness samples have the same structure as the indenters of the stiffness sensor, so that each indenter is in contact with the corresponding rod.

During the evaluation experiments, the stiffness sensor was fixed statically on the linear module. The stiffness sample were moved linearly towards the indenters. Forces, displacements, stiffness and contact angles were computed in realtime.

\section{A. Evaluation Test with stiffness samples}

Artificial stiffness samples with four different spring constants have been used for the experimental tests as shown in Fig. 4 The artificial samples were pushed against the stiffness sensor by $18 \mathrm{~mm}$ while recording the computed stiffness in real-time. The values of the measured stiffness and its distribution during one test performed with the artificial sample and a spring constant of $0.29 \frac{\mathrm{N}}{\mathrm{mm}}$ are shown in Fig. 6 The experimental results show that the proposed system is able to compute the stiffness of the springs embedded in the sample mechanism with high accuracy.

\begin{tabular}{||ccccc||}
\hline $\mathrm{K}$ & $K$ & $\sigma$ & RMSE & \% Accuracy \\
\hline 0.29 & 0.2908 & 0.012 & 0.0101 & 99.2393 \\
\hline 0.37 & 0.3812 & 0.0013 & 0.0113 & 98.8781 \\
\hline 0.62 & 0.6545 & 0.0346 & 0.0489 & 96.5466 \\
\hline 0.63 & 0.6674 & 0.0155 & 0.045 & 96.2610 \\
\hline
\end{tabular}

TABLE I: Evaluation test with the linear motor $\mathrm{K}$ represents the real stiffness of the springs used for the test, $\bar{K}$ it the mean of stiffness computed solving Equation (5), $\sigma$ and RMSE are the standard deviation and the root mean square. The last column shows the percentage of accuracy.

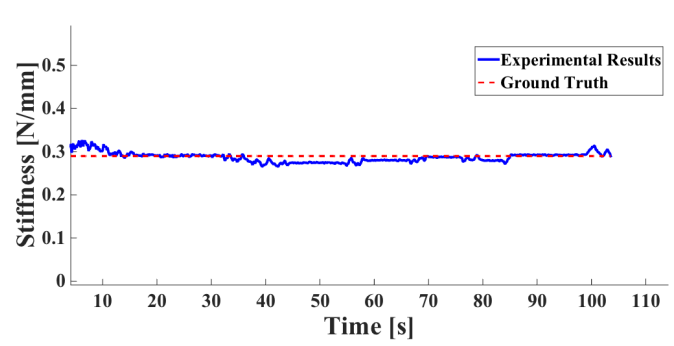

(a)

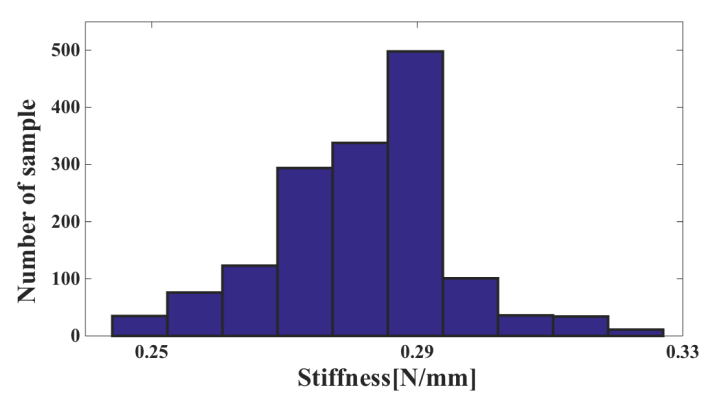

(b)

Fig. 5: Evaluation test for the artificial stiffness sample with a spring constant of $0.29 \frac{\mathrm{N}}{\mathrm{mm}}$ : (a) the measured stiffness and the ground truth are represented in blue and red respectively. (b) A histogram showing the distribution of the data for the above experiment.

Table II summarises the results obtained for all artificial stiffness samples. The error in the measurements increases with increasing stiffness of the evaluated spring constants. When interacting with "stiffer" objects, the difference in displacement between the "softer" and the "stiffer" springs inside the hand-held device descreases. Hence, the computation of the stiffness depends on the resolution of the camera which is used to track the spherical features.

\section{B. Evaluation Test with silicon}

In the previous section, VI-A we showed that the proposed sensor can compute the stiffness of a linear elastic material with high accuracy. In this section, we performed experimental tests using four silicon phantoms with different stiffness values. The material used were: Ooomoo ${ }^{\circledR} 30 \mathrm{~A}$, Dragon Skin ${ }^{\circledR} 20$ A, Ecoflex ${ }^{\circledR} 00-50$ and Ecoflex ${ }^{\circledR} 00-10$ by Smooth-On. Silicone is a non-linear elastic material. For small displacements, however, the response is approximately linear [24]. The test results in Fig. 6b show that our sensor is able to successfully distinguish materials with different stiffness levels even if the difference is relatively small as for example when comparing Dragon Skin ${ }^{\circledR} 20 \mathrm{~A}$ to Oodomo ${ }^{\circledR}$ $30 \mathrm{~A}$ - a scenario in which manual finger palpation fingers fails.

\section{Conclusions}

In this paper, a multi-axis stiffness sensor mechanism which relies on visual information has been presented. To the best of the authors' knowledge, this is the first system 


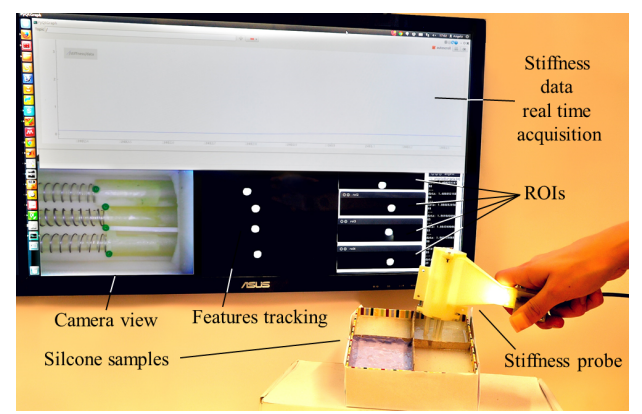

(a)

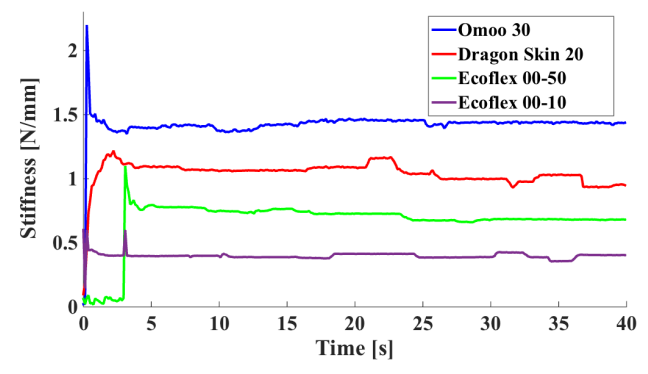

(b)

Fig. 6: Evaluation test with four different silicones:(a) experimental setup and (b) experimental results.

able to compute the stiffness independently of the orientation at which palpation is performed and to fully characterize the interaction with a soft surface. The sensing mechanism can be used for different medical applications. The working range and accuracy of the sensor can be adapted by replacing a spring with a different spring constant inside the hand-held device. If used for medical diagnosis, it can help clinicians to obtain adequate information about the presence of a mass inside a soft tissue organ without the need for X-ray - a method associated with health risks. Future development of the system will consider more evaluation with soft material, the integration of our sensing concept with an endoscopic camera and surgical tools for MIS.

\section{REFERENCES}

[1] T. GH, G. H, and R. CC, "Solitary thyroid nodule: Comparison between palpation and ultrasonography," Archives of Internal Medicine, vol. 155 , no. 22, pp. 2418-2423, 1995.

[2] T. O. Henderson, A. Amsterdam, S. Bhatia, M. M. Hudson, A. T. Meadows, J. P. Neglia, L. R. Diller, L. S. Constine, R. A. Smith, M. C. Mahoney, E. A. Morris, L. L. Montgomery, W. Landier, S. M. Smith, L. L. Robison, and K. C. Oeffinger, "Systematic review: Surveillance for breast cancer in women treated with chest radiation for childhood, adolescent, or young adult cancer," Annals of Internal Medicine, vol. 152 , no. 7, pp. 444-455, 2010.

[3] T. R. Coles, D. Meglan, and N. W. John, "The role of haptics in medical training simulators: A survey of the state of the art," IEEE Transactions on Haptics, vol. 4, no. 1, pp. 51-66, 2011.

[4] M.-S. Ju, H.-M. Vong, C.-C. Lin, and S.-F. Ling, "Development of soft tissue stiffness measuring device for minimally invasive surgery by using sensing cum actuating method," in 13th International Conference on Biomedical Engineering (C. Lim and J. Goh, eds.), vol. 23 of IFMBE Proceedings, pp. 291-295, Springer Berlin Heidelberg, 2009.

[5] M. van Veelen, E. Nederlof, R. Goossens, C. Schot, and J. Jakimowicz, "Ergonomic problems encountered by the medical team related to products used for minimally invasive surgery.," Surg Endosc, vol. 17, no. 7, pp. 1077-81, 2003.
[6] R. Muthupillai, D. Lomas, P. Rossman, J. Greenleaf, A. Manduca, and R. Ehman, "Magnetic resonance elastography by direct visualization of propagating acoustic strain waves," Science, vol. 269, no. 5232, pp. $1854-1857,1995$.

[7] Y. K. Mariappan, P. J. Rossman, K. J. Glaser, A. Manduca, and R. L. Ehman, "Magnetic resonance elastography with a phased-array acoustic driver system," Magnetic Resonance in Medicine, vol. 61, no. 3, pp. 678-685, 2009.

[8] E. H. Frei, B. D. Sollish, S. Yerushalmi, S. B. Lang, and M. Moshitzky, "Instrument for viscoelastic measurement," 1981.

[9] S. M. Hosseini, S. M. T. Kashani, S. Najarian, F. Panahi, S. M. M. Naeini, and A. Mojra, "A medical tactile sensing instrument for detecting embedded objects, with specific application for breast examination," The International Journal of Medical Robotics and Computer Assisted Surgery, vol. 6, no. 1, pp. 73-82, 2010.

[10] a. Sarvazyan, "Mechanical imaging: a new technology for medical diagnostics.," International journal of medical informatics, vol. 49, pp. 195-216, 1998.

[11] H. Liu, D. P. Noonan, B. J. Challacombe, P. Dasgupta, L. D. Seneviratne, and K. Althoefer, "Rolling mechanical imaging for tissue abnormality localization during minimally invasive surgery.," IEEE transactions on bio-medical engineering, vol. 57, pp. 404-14, Feb. 2010.

[12] J. Rosen, B. Hannaford, M. P. MacFarlane, and M. N. Sinanan, "Force controlled and teleoperated endoscopic grasper for minimally invasive surgery-experimental performance evaluation.," IEEE transactions on bio-medical engineering, vol. 46, pp. 1212-21, Oct. 1999.

[13] G.-P. Haber, M. A. White, R. Autorino, P. F. Escobar, M. D. Kroh, S. Chalikonda, R. Khanna, S. Forest, B. Yang, F. Altunrende, R. J. Stein, and J. H. Kaouk, "Novel robotic da vinci instruments for laparoendoscopic single-site surgery," Urology, vol. 76, no. 6, pp. 1279 $-1282,2010$.

[14] H. Liu, J. Li, X. Song, L. Seneviratne, and K. Althoefer, "Rolling indentation probe for tissue abnormality identification during minimally invasive surgery," Robotics, IEEE Transactions on, vol. 27, no. 3, pp. 450-460, 2011.

[15] H. Liu, P. Puangmali, D. Zbyszewski, O. Elhage, P. Dasgupta, J. S. Dai, L. Seneviratne, and K. Althoefer, "An indentation depthforce sensing wheeled probe for abnormality identification during minimally invasive surgery," Proceedings of the Institution of Mechanical Engineers, Part H: Journal of Engineering in Medicine, vol. 224, pp. 751763, June 2010.

[16] U. Seibold, B. Kubler, and G. Hirzinger, "Prototype of instrument for minimally invasive surgery with 6-axis force sensing capability," Robotics and Automation, no. April, pp. 498-503, 2005.

[17] P. Peng, a. S. Sezen, R. Rajamani, and a. G. Erdman, "Novel MEMS stiffness sensor for in-vivo tissue characterization measurement.," Conference proceedings: Annual International Conference of the IEEE Engineering in Medicine and Biology Society. IEEE Engineering in Medicine and Biology Society. Conference, vol. 2009, pp. 6640-3, Jan. 2009.

[18] A. P. Advincula and K. Wang, "Evolving role and current state of robotics in minimally invasive gynecologic surgery," Journal of Minimally Invasive Gynecology, vol. 16, no. 3, pp. 291 - 301, 2009.

[19] A. Faragasso, A. Stilli, J. Bimbo, Y. Noh, H. Liu, T. Nanayakkara, P. Dasgupta, H. A. Wurdemann, and K. Althoefer, "Endoscopic Addon Stiffness Probe for Real-time Soft Surface Characterisation in MIS," IEEE Engineering in Medicine and Biology Society (EMBC), 2014.

[20] A. Faragasso, J. Bimbo, Y. Noh, H. A. Wurdemann, S. Sareh, H. Liu, T. Nanayakkara, and K. Althoefer, "Novel Uniaxial Force Sensor based on Visual Information for Minimally Invasive Surgery," IEEE International Conference on Robotics and Automation (ICRA), no. Section V, 2014.

[21] M. I. a. Lourakis, "A Brief Description of the Levenberg-Marquardt Algorithm Implemened by levmar," Matrix, vol. 3, p. 2, 2005.

[22] D. W. Marquardt, "An algorithm for least-square estimation of nonlinear parameters," SIAM Journal on Applied Mathematics, vol. 11, no. $2,1963$.

[23] C. Laugier, C. Mendoza, and K. Sundaraj, "Towards a Realistic Medical Simulator using Virtual Environments and Haptic Interaction," In Proc. of the International Symposium in Reserach Robotics, Sidney (AU, 2001.

[24] Y. Fung, Biomechanics: Mechanical properties of living tissue. New York: Springer-Verlag, 1993. 